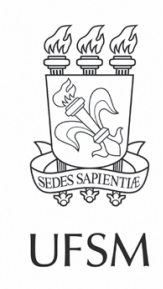

\title{
Artigos
}

\section{Monitoramento fenológico para avaliação de impacto ambiental em manguezais estuarinos no Nordeste do Brasil}

\author{
Phenological monitoring for environmental impact assessment in \\ estuarine mangroves in Northeastern Brazil
}

\author{
Jorgeana de Almeida Jorge Benevides ${ }^{\oplus}$ \\ Rafaela Camargo Maia" $\odot$ \\ Ingrid H'Oara Carvalho Vaz da Silva" ${ }^{\oplus}$
}

'Instituto Federal de Educação, Ciência e Tecnologia do Ceará, Paracuru, CE, Brasil

"Instituto Federal de Educação, Ciência e Tecnologia do Ceará, Acaraú, CE, Brasil

\section{RESUMO}

Os manguezais se configuram em um dos ecossistemas mais produtivos do planeta, entretanto eles vêm sofrendo progressivas alterações ambientais, devido às pressões antrópicas. Informações sobre o comportamento fenológico podem ser utilizadas como instrumento na construção de projetos de conservação de recursos naturais. O presente estudo foi realizado em quatro áreas estuarinas em Acaraú, Ceará, objetivando analisar o comportamento fenológico dos componentes reprodutivos de três espécies de mangue em diferentes áreas sujeitas a diferentes tipos de impactos ambientais (resíduos sólidos, desmatamento e efluentes da carcinicultura) e uma área de baixo impacto no período de um ano. A fim de se caracterizar os padrões fenológicos foi realizada observação direta, registrandose a presença e a intensidade da floração e frutificação. Dois métodos de análise foram aplicados para entender os padrões de floração e frutificação: percentual de intensidade de Fournier e o índice de atividade. As florações de Avicennia spp. e Rhizophora mangle L, em condições naturais, ocorrem o ano todo, e nas áreas impactadas mostraram padrões irregulares. Quanto à frutificação, Laguncularia racemosa (L.) C. F. Gaertn foi a espécie que apresentou os mais altos percentuais para os dois índices fenológicos analisados. A pluviosidade e a salinidade parecem exercer maior força seletiva quando as espécies estão sujeitas a algum tipo de impacto antrópico. A análise comparativa dos diferentes métodos de avaliação fenológicos realizados neste estudo indicou que estes fornecem dados distintos e complementares sobre o comportamento reprodutivo vegetal e de como os impactos a que estes ambientes estão sujeitos pode estar interferindo negativamente sobre a flora deste ecossistema.

Palavras-chave: Fenologia; Mangue; Pressão antrópica 


\begin{abstract}
Mangroves are one of the most productive ecosystems on the planet however they have undergone progressive environmental changes due to anthropic pressures. Information on phenological behavior can be used as an instrument in the construction of conservation projects of natural resources. The present study was carried out in four estuarine areas in Acaraú, Ceará, Brazil, with the aim of analyzing of phenological behavior regarding reproductive components of three mangrove species in different areas subject to different environmental impacts (solid waste, deforestation and shrimp farm effluent) and a low impacted area, during a period of one year. patterns of flowering and fruiting: Fournier Index (percentage of intensity) and the index of activity. The flowering of Avicennia spp. and Rhizophora mangle, under natural conditions, occurred all year round, and it showed irregular patterns. Regarding fruiting, Laguncularia racemosa was the species that presented the highest values for the two phenological indices analyzed. Rainfall and salinity seemed to exert a more selective force when species were subjected to an anthropic impact. The comparative analysis of the different phenological evaluation methods carried out in this study indicated that they provide different and complementary data about the reproductive behavior of the plant and how the impacts to which these environments are subjected may be negatively interfering with the flora of this ecosystem.
\end{abstract}

Keywords: Phenology; Mangrove; Antropic pressure

\title{
1 INTRODUÇÃO
}

O mangue é uma vegetação que se encontra em áreas de transição entre os ambientes terrestre e marinho. Os manguezais estão cada vez mais sujeitos à degradação decorrente das atividades humanas (BONALDI; RODERJAN, 2017) e o monitoramento ambiental apresenta-se como uma ferramenta viável, considerando os potenciais impactos ambientais decorrentes da pressão antrópica negativa sobre os manguezais. O acompanhamento sistemático do panorama ecológico permite a criação de políticas públicas visando à recuperação e à melhoria da qualidade ambiental. Os manguezais estão amparados legalmente devido a sua importância ecológica (e.g. sequestro de carbono, renovação da biomassa costeira) (FERREIRA; BEZERRA; MATTHEWS-CASCON, 2019), econômica (e.g. pesca artesanal) e a alta suscetibilidade à poluição dos solos e dos recursos hídricos. Entretanto, o avanço das pressões antrópicas sobre os manguezais em todo mundo vem causando perda de habitats assim como interferindo em seus bens e serviços ecossistêmicos, como, por exemplo, o de conectividade. 
O despejo de resíduos sólidos urbanos representa um grave problema socioambiental. O descarte costuma se concentrar em áreas estuarinas ou em águas marinhas costeiras. Essa prática acaba interferindo na estrutura, fisiologia e biodiversidade desse ecossistema (MATIAS; SILVA, 2017). O avanço progressivo da especulação imobiliária sobre as áreas costeiras também se configura como um tensor e uma das consequências é o desmatamento. Em manguezais desmatados, observouse que a retirada de madeira pode alterar os valores de produtividade, uma vez que modifica a estrutura e a composição florística (PAULA et al., 2016). Estas mesmas autoras também apontam a carcinicultura como uma das razões para a degradação do manguezal.

Pesquisas que permitam um melhor entendimento da dinâmica entre as espécies da comunidade de mangue servirão como subsídio para futuras intervenções na tentativa de recuperação das áreas impactadas. Nesse contexto, o presente trabalho visou analisar o comportamento fenológico de mangue como instrumento de avaliação de impacto ambiental, considerando o grau de perturbação antrópica nesse ambiente estuarino.

\section{MATERIAL E MÉTODOS}

Esta pesquisa foi desenvolvida no manguezal do estuário do rio Acaraú (0249'94" S, 4005'14" O) na costa ocidental do Ceará, no nordeste do Brasil. A temperatura média da região é de $27^{\circ} \mathrm{C}$ e a pluviosidade aproximada de $1100 \mathrm{~mm}$ ao ano. As chuvas mais significativas na região têm início em dezembro podendo se estender até julho (FUNDAÇÃO CEARENSE DE METEOROLOGIA E RECURSOS HÍDRICOS, 2016).

No município de Acaraú foram escolhidas quatro áreas sob as mesmas condições ambientais (Figura 1), porém sujeitas a diferentes pressões antrópicas resultantes da carcinicultura, disposição inadequada de resíduos sólidos e desmatamento e uma com baixo nível de impacto ambiental (área controle). As áreas foram selecionadas após análise das imagens de satélite da região e visitas aos manguezais. 
Figura 1 - Mapa com a localização das áreas de estudo, Acaraú, Brasil. Em destaque os pontos de coleta nos manguezais analisados

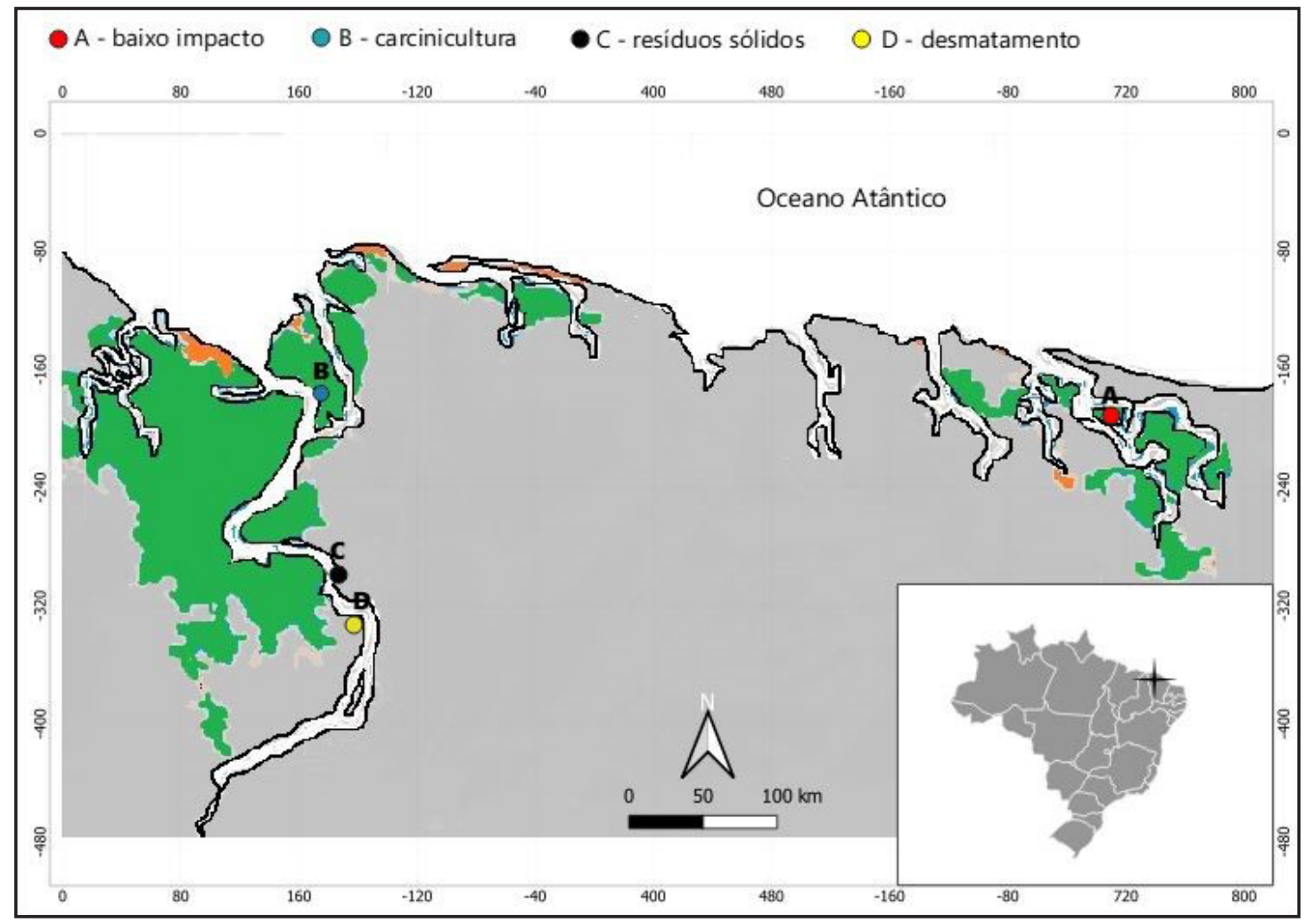

Fonte: Autoras (2016)

Em que: A - baixo impacto; B - carcinicultura; C - resíduos sólidos; D - desmatamento.

Durante as amostragens também foram registradas as temperaturas da água, do ar, do sedimento e umidade relativa com ajuda de um termohigrômetro e verificada a salinidade com um refratômetro. Os dados de pluviosidade foram obtidos junto à Fundação Cearense de Meteorologia e Recursos Hídricos - anos 2015/2016.

A fim de caracterizar o padrão fenológico de floração e frutificação, foram escolhidos ao acaso 15 indivíduos de cada espécie em cada área, em vários estágios de maturação, da flora de mangue mais representativas na região (Avicennia germinans, Avicennia schaueriana, Laguncularia racemosa e Rhizophora mangle). A baixa ocorrência de espécimes aptos para a realização desta pesquisa nos fez agrupar as espécies Avicennia germinans e Avicennia schaueriana. Tendo em vista a limitação no quantitativo de indivíduos em cada local, na área impactada por desmatamento não foi monitorada a espécie Rhizophora mangle. Totalizando assim 165 espécimes monitorados nas quatro áreas amostrais. 
Mensalmente (abril/2015 a março/2016), no período diurno foram realizadas observações, com auxílio de binóculo, registrando-se a presença das fenofases floração e frutificação, por indivíduo. Cada planta foi identificada e etiquetada por área. Os dados obtidos foram utilizados para determinar: i) a presença e intensidade da floração (emissão de flores femininas e masculinas); ii) a presença e a intensidade da frutificação. Como critério para definição de floração utilizou-se a época em que as árvores exibem botões florais ou flores; e para frutificação (propágulos imaturos e maduros), fase em que eles se encontraram fixos à planta-mãe.

Dois métodos de análise foram aplicados na tentativa de entender os padrões de floração e frutificação: percentual de intensidade de Fournier (1974) e o índice de atividade (BENCKE; MORELLATO, 2002a). Eles fornecem dados distintos e complementares sobre respostas fenológicas em amostras populacionais (BENCKE; MORELLATO, 2002a).

Por meio do índice de atividade, pode-se também estimar o sincronismo dentro da população amostrada. Em relação ao índice de atividade, foram obedecidos os valores percentuais sugeridos no trabalho de Morellato et al. (1990), o padrão fenológico de floração e frutificação das espécies foram interpretados e analisados para a determinação de parâmetros de início, pico e término das fenofases reprodutivas dos três gêneros de plantas testadas assim como a sincronia dos eventos. A seguir, foi realizada uma análise descritiva dos parâmetros analisados e foram testadas a normalidade (Shapiro Wilk e Kolmogorov-Smirnov) e a homogeneidade dos dados (Teste de Levene).

Foi realizada uma Análise de Correlação linear entre os parâmetros abióticos (umidade, salinidade, temperatura e pluviosidade) e bióticos (percentual de intensidade de Fournier, índice de atividade - presença de flor e/ou fruto, produção total e frações de flor e frutos). Para subsidiar a seleção dos descritores utilizados nessa análise, utilizou-se uma correlação entre todas as variáveis, retirando das análises os parâmetros altamente correlacionados $(R>0,95)$. Todas as análises foram realizadas utilizando o programa STATISTICA for Windows® versão trial. 


\section{RESULTADOS}

Em relação aos valores de temperatura do ar, a mínima foi de $24,5^{\circ} \mathrm{C}$ (março/2016) e máxima de $37^{\circ} \mathrm{C}$ (junho/2015), na área de carcinicultura. As áreas de resíduos sólidos e desmatamento exibiram valores de temperatura mais elevados que nas outras duas áreas durante todo o período. Nas áreas de carcinicultura e baixo impacto foram registradas as maiores médias para os percentuais de umidade do ar entre as áreas estudadas (70\% e 67\%, respectivamente). Na área desmatada a média foi de $64 \%$ e 60\% na área de resíduos sólidos.

O período de agosto a novembro de 2015 configurou-se como os meses secos, uma vez que nenhuma pluviosidade foi registrada neste período (Figura 2).

Figura 2 - Relação entre os valores mensais de salinidade do rio nas áreas de baixo impacto (BI), carcinicultura (CARC), resíduos sólidos (RES) e desmatamento (DESM) e o total de chuvas acumulado (mm) entre abril de 2015 e março de 2016

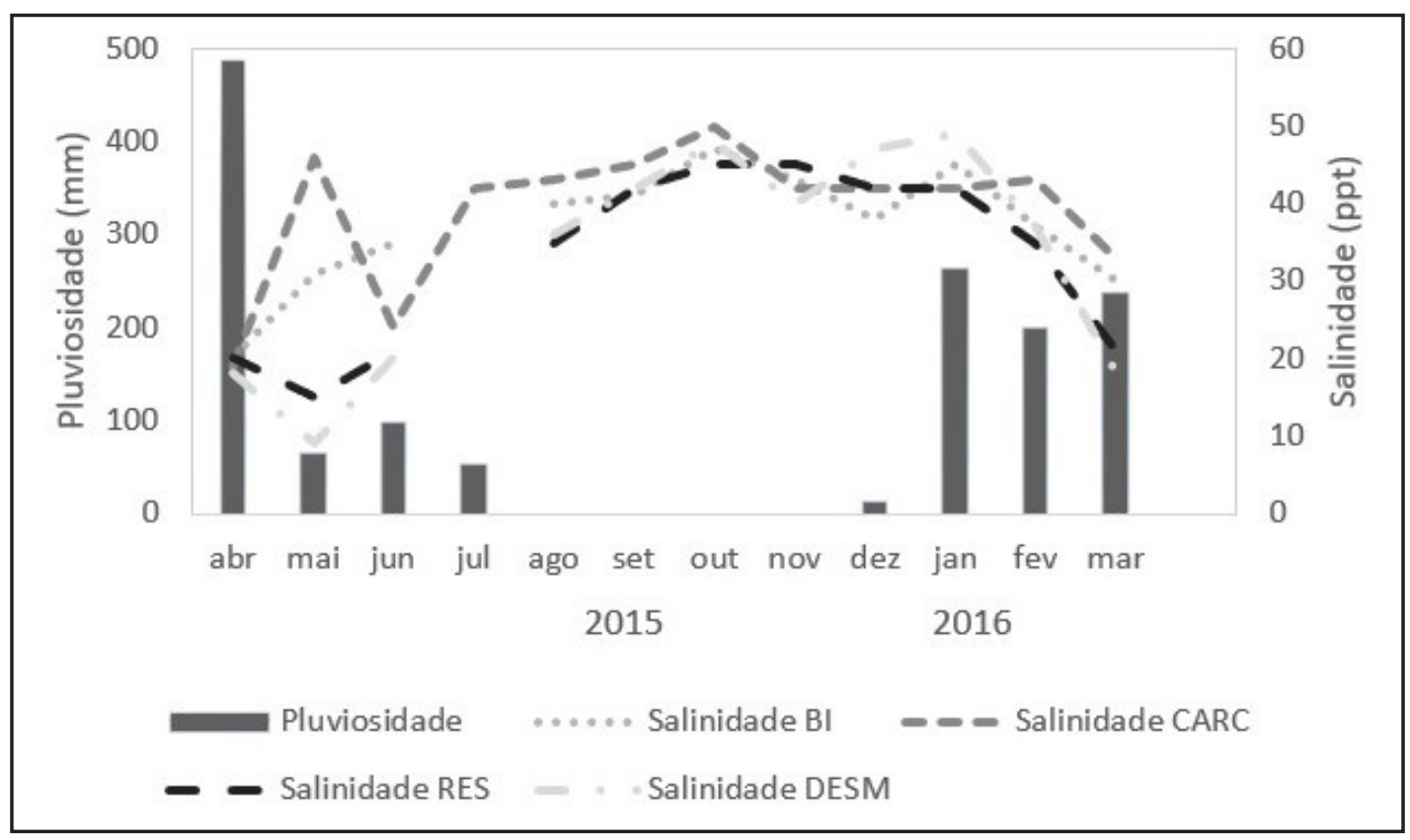

Fonte: Autoras (2016)

Em que: *A barra refere-se à pluviosidade na região de estudo e as linhas as taxas de salinidade nas quatro áreas. Não foi possível mensurar os dados de julho/2015 nas áreas de baixo impacto, resíduos sólidos e desmatamento. 
O pico de chuva ocorreu em abril, com máxima observada de $486,7 \mathrm{~mm}$ (FUNDAÇÃO CEARENSE DE METEOROLOGIA E RECURSOS HÍDRICOS, 2016). A partir desse mês houve declínio até o mês de julho e novas precipitações a partir de dezembro (Figura 2). A máxima taxa de salinidade foi registrada em janeiro/2016 na área de desmatamento (49). A área influenciada pela carcinicultura registrouse os valores de salinidade mais elevados na maioria dos meses de estudo. Não foi possível obter os dados abióticos relativos ao mês de julho/2015 em três áreas (baixo impacto, resíduos e desmatamento).

Tanto nos meses secos quanto nos chuvosos foram observados valores elevados para salinidade (>40 ppm nas quatro áreas), contudo houve um decréscimo mais acentuado a partir de fevereiro/2016 nas áreas de baixo impacto, resíduos sólidos e desmatamento (Figura 2).

Quando analisada a atividade de floração das espécies entre as áreas, verificouse que nas áreas impactadas por carcinicultura, resíduos sólidos e desmatamento, Avicennia spp apresentou floração em sete dos doze meses avaliados (Figura 3A).

A atividade de floração ocorreu tanto nos meses secos quanto nos chuvosos. Apesar de apresentarem atividade em outros meses do ano, nos meses entre outubro/2015 a janeiro/2016, Avicennia spp. apresentou atividade de floração altamente sincrônica entre os indivíduos da população e, coincidentemente, entre as áreas de baixo impacto, resíduos sólidos e desmatamento (Figura 3A). Ou seja, nesse período mais de $60 \%$ dos indivíduos da população apresentaram a mesma fenofase ao mesmo tempo nas três áreas. Dentre os impactos, a população de Avicennia spp. parece ter sido mais afetada na área de carcinicultura, pois dos sete meses de atividade, seis foram de baixo sincronismo entre os indivíduos amostrados (período de agosto/2015 a janeiro/2016). Em fevereiro/2016 a atividade foi assincrônica (<20\%).

Nas áreas impactadas, a floração de Avicennia spp. não se apresentou de forma contínua como ocorreu na área de baixo impacto. Embora na área controle a floração tenho sido mais prolongada, nas áreas de resíduos sólidos e desmatamento os índices de sincronia foram altos de outubro/2015 a janeiro/2016 (taxas entre $81 \%$ a 100\%). 
Figura 3 - Índice de Atividade (ou porcentagem de indivíduos) por espécie e por áreas para a fenofase flor e fruto em manguezais estuarinos do Rio Acaraú, Ceará

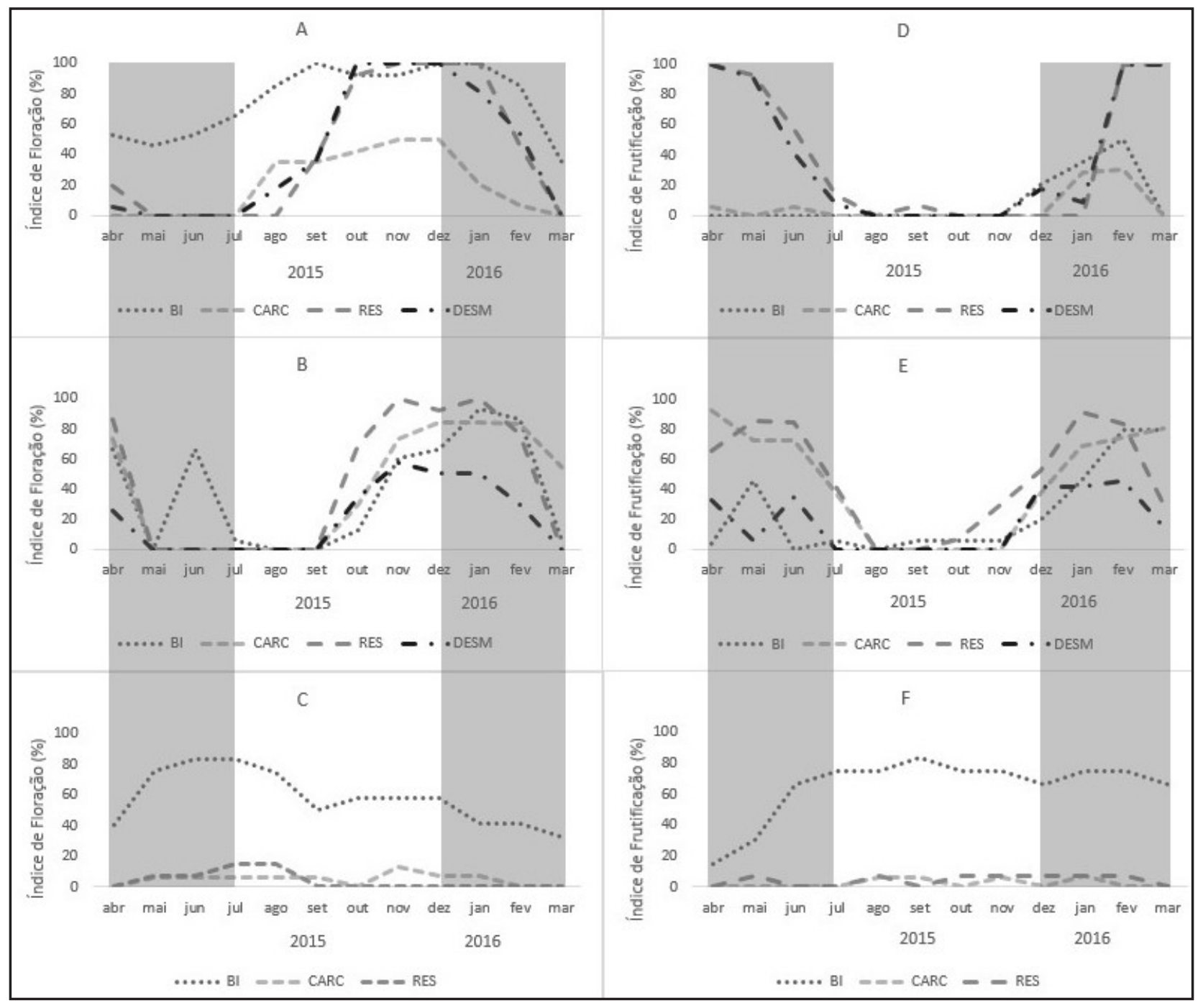

Fonte: Autoras (2016)

Em que: *As áreas hachuradas indicam os meses chuvosos durante o período de estudo; A - Índice de Floração de Avicennia spp.; B - Índice de Floração de Laguncularia racemosa; C - Índice de Floração de Rhizophora mangle; D - Índice de Frutificação de Avicennia spp.; E - Índice de Frutificação de Laguncularia racemosa; F - Índice de Frutificação de Rhizophora mangle.

Em relação à Laguncularia racemosa, nas três áreas impactadas os indivíduos monitorados exibiram atividade de floração similar quanto à época de ocorrência. $\mathrm{Na}$ área de baixo impacto (Figura 3B), a floração ocorreu entre abril/2015 e julho/2015, com exceção do mês de maio. Depois voltando à atividade entre outubro do mesmo ano a março de 2016, com alto sincronismo entre novembro/2015 e fevereiro/2016, 
reforçando que Laguncularia racemosa não exibe produção de flores contínua ao longo do ciclo anual.

$\mathrm{Na}$ área de carcinicultura poucos indivíduos de Rhizophora mangle apresentaram atividade de floração, com porcentagem variando entre 6\% e 13\% indivíduos por oito meses dos 12 avaliados (Figura 3C). Enquanto a área de resíduos sólidos a espécie apresentou entre $7 \%$ e $15 \%$ de indivíduos em atividade concentrados entre os meses de maio/2015 e agosto/2015. Contrário ao que ocorreu nas áreas impactadas, na área de baixo impacto Rhizophora mangle florou o ano todo com altos índices de sincronismo entre maio/2015 e agosto/2015.

Em relação ao índice de atividade para a fenofase frutificação, as três espécies apresentaram número inferior de percentual de indivíduos manifestando a fenofase de frutificação em relação aos de floração.

Avicennia spp. apresentou porcentagens mais elevadas de indivíduos manifestando a fenofase de frutificação nas áreas de resíduos sólidos e desmatamento (Figura 3D). Nos meses de abril/2015, maio/2015, fevereiro/2016 e março/2016, meses chuvosos, os indivíduos de Avicennia spp. monitorados nessas duas áreas apresentaram alta sincronia quanto à frutificação.

$\mathrm{Na}$ área de carcinicultura os índices de atividade ocorreram em quatro meses dos 12 avaliados. Os casos de sobreposição das fenofases floração e frutificação da Avicennia spp. ocorreram de formas pontuais nas quatro áreas. $\mathrm{Na}$ área desmatada, a sobreposição das fenofases ocorreram nos meses de abril/2015 e entre dezembro/2015 a fevereiro/2016. Na área de resíduos sólidos foi registrada sobreposição nos meses de abril/2015 e setembro/2015. Em carcinicultura não foi registrada sobreposição das fenofases reprodutivas.

Embora na área de baixo impacto a floração tenha se estendido durante todo o período de estudo, a frutificação ocorreu concomitantemente somente nos meses de dezembro/2015 a fevereiro/2016. Nas áreas impactadas pela carcinicultura e por resíduos sólidos, Laguncularia racemosa apresentou alto sincronismo para frutificação 
entre abril/2015 e junho/2015, e em 2016 nos meses de janeiro e fevereiro, chuvosos na região.

Na área desmatada os indivíduos apresentaram percentuais entre 6\% e 46\% de frutificação para a espécie Laguncularia racemosa, demonstrando períodos de baixa sincronia e assincrônicos (Figura 3E).

Nas áreas impactadas não foi observada a mesma recorrência de sobreposição das fenofases de Rhizophora mangle como foi verificada na área de baixo impacto durante os 12 meses de pesquisa (Figura 3F). Também foi constatado um baixo índice de frutificação de Rhizophora mangle nas áreas impactadas.

Nas áreas de baixo impacto, carcinicultura e resíduos sólidos, a floração de Laguncularia racemosa exibiu alto sincronismo para a fenofase flor na transição dos meses secos para o chuvoso. E nas áreas de carcinicultura e resíduos sólidos foram registrados os melhores percentuais de floração e frutificação para Laguncularia racemosa (Figuras 3B e 3E).

Nas áreas impactadas, o período de floração de Laguncularia racemosa mais intenso se concentrou entre os meses de outubro/2015 a março/2016. No tocante à frutificação entre estas mesmas áreas foram observadas pausas ou diminuição na atividade nos meses sem chuva.

Nas áreas de carcinicultura e resíduos sólidos foi observado assincronismo tanto para floração quanto para frutificação de Rhizophora mangle. Na área de baixo impacto, a floração e frutificação desta espécie apresentou alto sincronismo ao longo de quatro e dez meses, respectivamente (Figura 4A).

Os percentuais de intensidade para floração e frutificação de Avicennia spp., Laguncularia racemosa e Rhizophora mangle são em sua maioria inferiores aos percentuais de atividade; isso significa que há alta sincronia entre os indivíduos dessas espécies, porém baixa intensidade de floração. 
Figura 4 - Índices de Intensidade para floração e frutificação nas áreas de baixo impacto (A), carcinicultura (B), resíduos sólidos (C) e desmatamento (D)

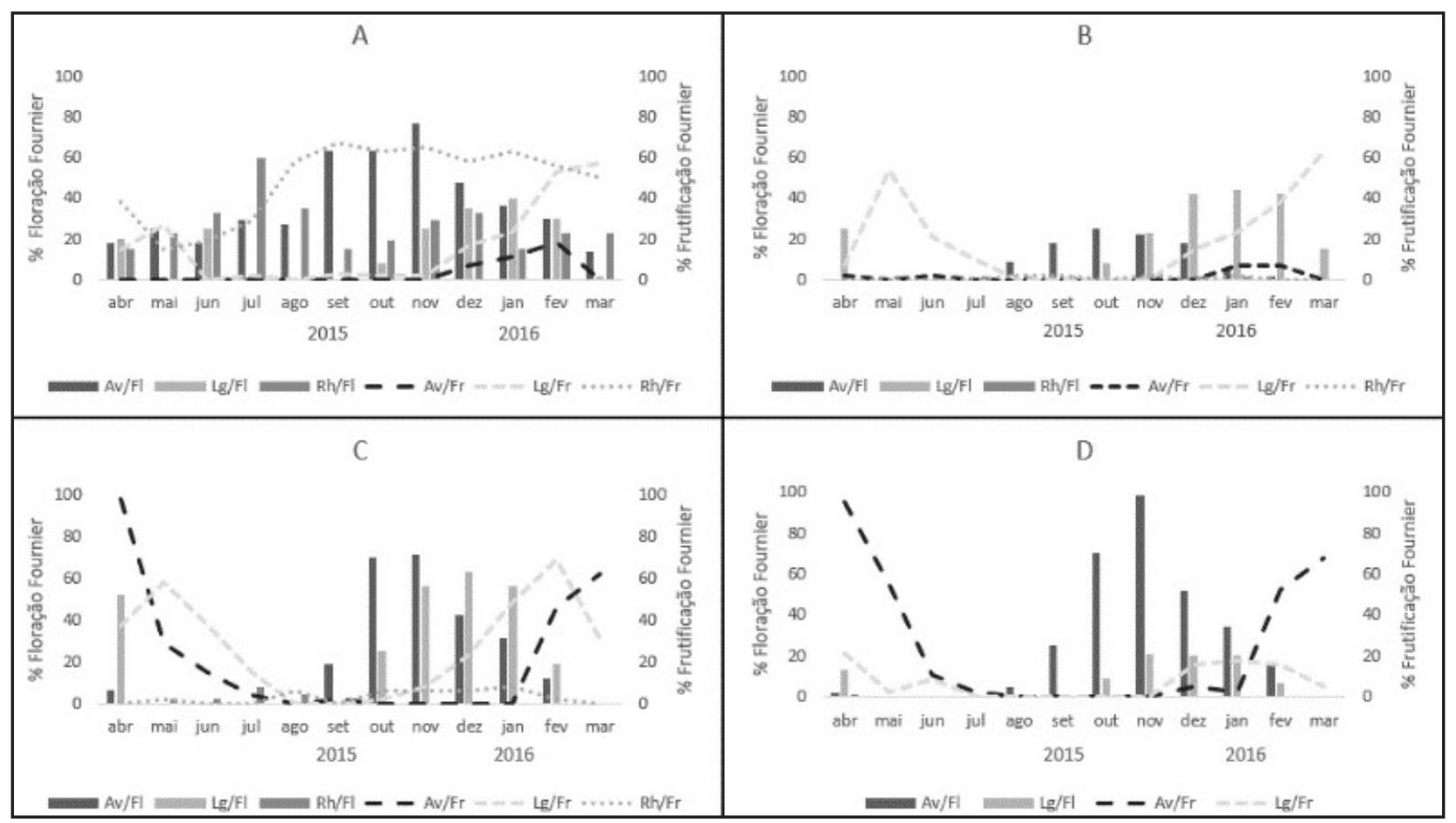

Fonte: Autoras (2016)

Em que: *As barras representam a fenofase floração (FI); as linhas, a fenofase frutificação (Fr); Av: Avicennia; Lg: Laguncularia; Rh: Rhizophora.

O gênero Avicennia apresentou na área de carcinicultura os mais baixos percentuais de intensidade ( $\leq 25 \%$ ) para floração (Figura 4B). Registros de alta intensidade de floração foram observados nas áreas de resíduos sólidos e desmatamento (Figura 4C e 4D) nos meses outubro/2015 e novembro/2015, final do período de estiagem na região. Na área controle foi registrado pico de intensidade de floração para Avicennia spp. no mês de novembro/2015 (Figura 4A).

Picos de atividade e de intensidade podem coincidir como ocorreu com a floração Laguncularia racemosa na área de resíduos sólidos (Figura 4C) nos meses de novembro/2015 e janeiro/2016, mas sua interpretação nem sempre é a mesma como no caso da floração de Avicennia spp. nos meses de outubro/2015 e novembro/2015 na área de desmatamento. Ressalta-se que tanto os índices de atividade (100\%), como os percentuais de intensidade foram altos (70\% e 98\%, respectivamente) para 
Avicennia spp., enquanto que no caso de Laguncularia racemosa, esta apresentou índices de 100\% de atividade, mas em relação à intensidade os resultados foram $56 \%$ para ambos os índices avaliados. Ou seja, mesmo que os picos coincidam, eles podem expressar valores bem distintos

A única área que apresentou percentuais altos em relação à intensidade de floração de Laguncularia racemosa foi área com resíduo sólido nos meses de abril/2015, novembro/2015 a janeiro/2016. Nas áreas impactadas foram registrados baixos percentuais de intensidade de floração para Rhizophora mangle.

Quanto ao percentual de intensidade de frutificação de Avicennia spp., nas áreas impactadas por resíduos sólidos e desmatamento foram observados picos intensidade na produção de frutos no mês mais chuvoso do período de estudo (abril/2015), mas o mesmo padrão não foi manifestado pela espécie nas áreas de carcinicultura e baixo impacto. Nessas duas áreas foram registrados os mais baixos índices para esta fenofase.

A intensidade da frutificação em Laguncularia racemosa foi a melhor entre as três espécies, mas apresentou percentuais, de modo geral, inferiores aos de atividade. Quando comparados ao percentual de intensidade no mesmo mês e área, o resultado foi de $7 \%$, concluiu-se então que os métodos analisados neste estudo forneceram informações essencialmente distintas como dito anteriormente. Na área desmatada (Figura 5) os índices de intensidade foram baixos ( $\leq 21 \%$ ). Os altos percentuais de frutificação foram registrados nos meses com chuva como se deu no mês de fevereiro/2016 na área de resíduos sólidos (Figura 5) e em março/2016 nas áreas de carcinicultura e baixo impacto.

Nas áreas impactadas, a espécie Rhizophora mangle exibiu baixos percentuais quanto à intensidade de frutificação ( $\leq 8 \%)$. Na área de baixo impacto (Figura 4 ), a espécie apresentou níveis distintos de intensidade (entre 15\% e 67\%) durante todo o período de estudo sugerindo que de alguma forma os tensores antrópicos estão interferindo na intensidade desta fenofase em Rhizophora mangle. Os picos de intensidade e de atividade de frutificação desta espécie coincidiram e ocorreram em setembro/2015, mês sem registro de chuva. 
Figura 5 - Fenogramas com percentuais de intensidade de floração e frutificação nas áreas de baixo impacto (BI), de carcinicultura (CARC), de resíduos sólidos (RES) e desmatamento (DESM)

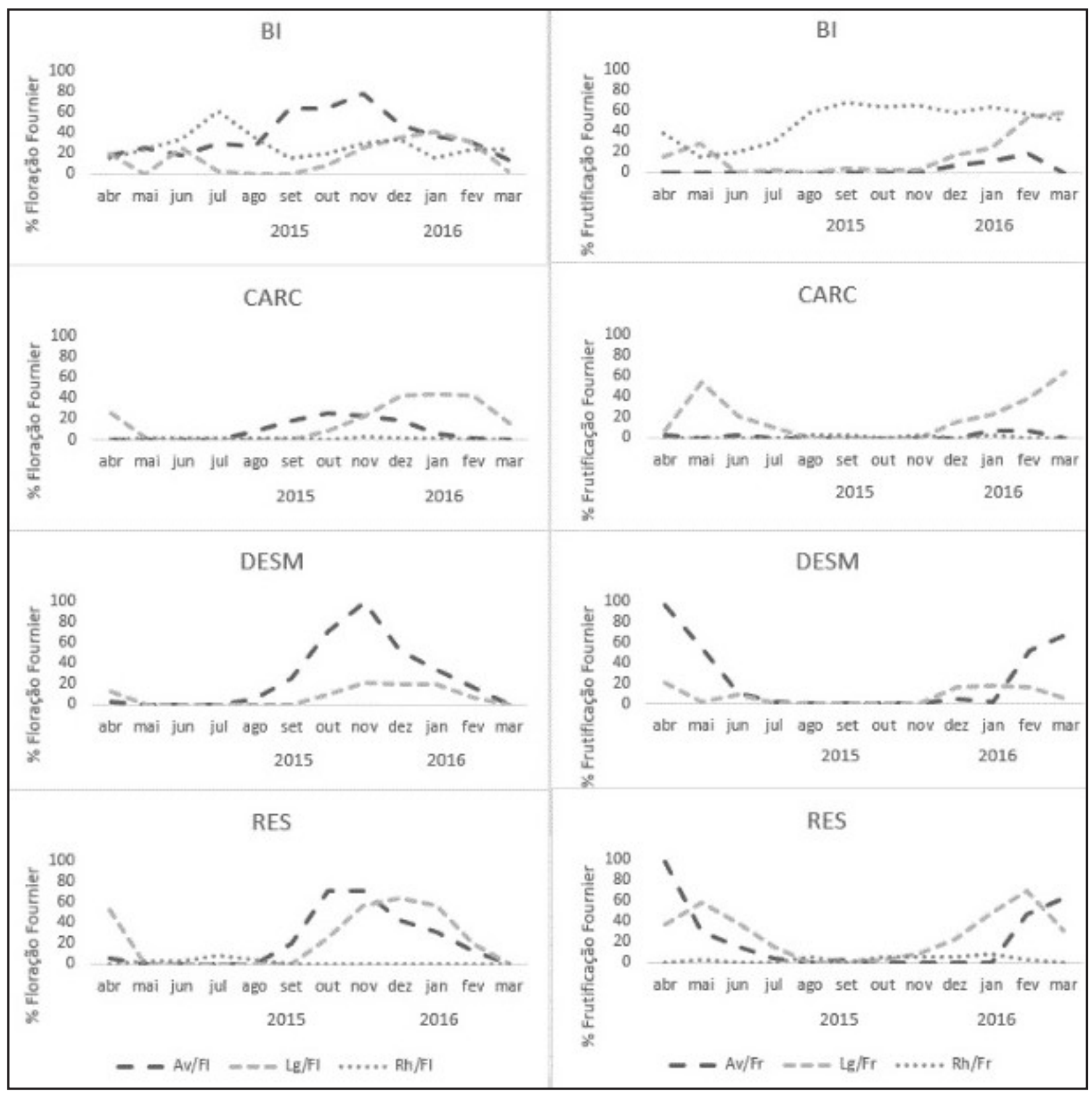

Fonte: Autoras (2016)

Em que: Av - Avicennia spp, floração e frutificação; Lg - Laguncularia racemosa, floração e frutificação; Rh - Rhizophora mangle, floração e frutificação.

Os dados comparativos ao longo dos 12 meses revelaram que os indivíduos das áreas impactadas apresentaram menos atividade que em relação aos da área de baixo 
impacto, especialmente em relação à atividade de floração e frutificação de Rhizophora mangle. Nos meses de maio/2015 a julho/2015 e março/2016, nas áreas impactadas, não foram registrados indivíduos de Avicennia spp. em atividade diferentemente do que ocorreu na área de baixo impacto.

O resultado da Análise de Correlação entre a fenologia e os dados abióticos demonstraram diferentes respostas entre as áreas analisadas $(p<0,05)$. Na área de carcinicultura a frutificação, tanto a sua atividade quanto a sua intensidade, apresentou forte correlação com a pluviosidade (Tabela 1). Na área afetada por resíduos sólidos, a frutificação também revelou correlação positiva com a pluviosidade; sendo uma moderada correlação em relação à atividade e forte correlação com a intensidade da frutificação. Ainda nesta área foram observadas correlações entre a atividade e a intensidade de floração com a salinidade.

A área desmatada foi a única a apresentar correlação entre a frutificação e a umidade. Nesta área, a atividade e intensidade de floração apresentaram forte correlação positiva com a salinidade. A atividade e a intensidade de frutificação manifestaram correlação positiva com a pluviosidade.

Na área de baixo impacto não foi observada correlação com fatores abióticos.

Nos meses secos houve um decaimento na produção de frutos nas três áreas impactadas. Na área de baixo impacto, observou-se que as florações de Avicennia spp. e Rhizophora mangle foram contínuas ao longo do ano de estudo, o que não ocorreu nas áreas sujeitas a impactos antrópicos.

Os níveis de salinidade flutuaram bastante ao longo dos meses nas quatro áreas (Figura 1). Destaque para a atividade e a intensidade da floração que apresentaram correlação positiva em relação à salinidade em duas áreas impactadas (resíduos sólidos e desmatamento). 
Tabela 1 - Resultado da Análise de Correlação entre as fenofases reprodutivas e os dados abióticos (umidade, salinidade, temperatura do ar e pluviosidade) nas quatro áreas estudadas

\begin{tabular}{|c|c|c|c|c|}
\hline Área & Umidade & Salinidade & Temp. Ar & Pluviosidade \\
\hline \multirow[t]{2}{*}{ Variáveis } & Uimuaue & Samiluade & 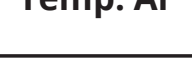 & riuviosituatue \\
\hline & & & & $\mathrm{BI}$ \\
\hline Flor Atividade & $-0,01$ & 0,35 & 0,16 & $-0,08$ \\
\hline Fruto & 0,28 & 0,14 & 0,05 & 0,47 \\
\hline Flor Intensidade & $-0,39$ & 0,26 & $-0,05$ & $-0,46$ \\
\hline \multirow[t]{2}{*}{ Fruto Intensidade } & 0,29 & 0,40 & 0,25 & 0,25 \\
\hline & & & & CARC \\
\hline Flor Atividade & $-0,11$ & 0,15 & $-0,51$ & 0,08 \\
\hline Fruto & 0,42 & $-0,55$ & 0,48 & 0,80 \\
\hline Flor Intensidade & $-0,07$ & 0,23 & $-0,48$ & 0,07 \\
\hline \multirow[t]{2}{*}{ Fruto Intensidade } & 0,50 & $-0,56$ & 0,35 & 0,83 \\
\hline & & & & RES \\
\hline Flor Atividade & 0,03 & 0,73 & 0,27 & 0,04 \\
\hline Fruto & 0,24 & $-0,34$ & 0,22 & 0,66 \\
\hline Flor Intensidade & 0,07 & 0,65 & 0,26 & $-0,05$ \\
\hline \multirow[t]{2}{*}{ Fruto Intensidade } & 0,29 & $-0,27$ & 0,26 & 0,84 \\
\hline & & & & DESM \\
\hline Flor Atividade & 0,21 & 0,85 & 0,25 & $-0,26$ \\
\hline Fruto & 0,58 & $-0,27$ & 0,22 & 0,63 \\
\hline Flor Intensidade & 0,10 & 0,74 & 0,28 & $-0,35$ \\
\hline Fruto Intensidade & 0,47 & $-0,31$ & 0,17 & 0,71 \\
\hline
\end{tabular}

Fonte: Autoras (2016)

Em que: *Os valores em negrito indicam correlações significativas ( $p<0,05)$; Baixo Impacto - Bl; Carcinicultura - CARC; Resíduos Sólidos - RES; Desmatamento - DESM; Temp. - temperatura.

\section{DISCUSSÃO}

As áreas de resíduos sólidos e desmatamento exibiram valores de temperatura mais elevados quando comparadas às outras duas áreas (médias anuais de $32^{\circ} \mathrm{C} \mathrm{e}$ 
$31^{\circ} \mathrm{C}$, respectivamente). A presença de clareiras na área de desmatamento e a menor densidade de árvores devido à mortalidade provocadas pelo acúmulo de resíduos sólidos podem contribuir para esse resultado (PAULA et al., 2016). No entanto, os resultados da Análise de Correlação não apresentaram correlações significativas entre a temperatura e as fenofases analisadas. O mesmo ocorreu em relação à umidade (Tabela1).

A reprodução e o crescimento vegetativo das plantas podem ser influenciados por fatores como a sazonalidade climática. Entretanto, há variações quanto aos principais fatores climáticos que realmente vão exercer mudanças nos padrões fenológicos em comunidades vegetais, no presente trabalho representado por variações de pluviosidade e salinidade (ALVARENGA; BOTOSSO; SOFFIATTI, 2017).

A atividade de floração da Avicennia spp. ocorreu tanto nos meses secos quanto nos chuvosos, sugerindo que a chuva pode não ser o fator mais importante na indução da reprodução nesta espécie. As plantas arbóreas de florestas tropicais podem apresentar fenofases reprodutivas bimodal, ou seja, com picos no período chuvoso e seco (OCHOA-GAONA; HERNÁNDEZ; JONG, 2008). Isso parece ser importante para ambientes fragmentados, pois de acordo com Ferreira e Consolaro (2013), a presença de espécies em floração e frutificação auxiliam na manutenção de polinizadores e dispersores em fragmento, contribuindo para a dinâmica e permanência da comunidade, por menor que seja a área ou a riqueza de espécies.

O abreviamento observado na atividade de floração nas áreas impactadas pode ser uma resposta aos respectivos impactos, uma vez que o padrão de floração de Rhizophora mangle foi anual e contínuo na área controle. A literatura ressalta que dentre as espécies de Rhizophoraceae, as respostas fenológicas podem estar relacionadas a fatores endógenos, como também podem ser alteradas por fatores ambientais para a espécie Rhizophora mangle (ALVARENGA; BOTOSSO; SOFFIATTI, 2017; LIMA, 2018).

Os casos de sobreposição de fenofases reprodutivas não foram frequentes. Isso pode estar relacionado com a tentativa da planta de evitar sobrecarga de recursos 
energéticos ao limitar o tempo que essas duas fenofases ocorram ao mesmo tempo (FERREIRA; CONSOLARO, 2013).

Laguncularia racemosa apresentou alto sincronismo para frutificação dentro dos meses chuvosos na região. Segundo Sousa et al. (2007), esse resultado pode se configurar como uma adaptação ao ambiente inundado, uma vez que permite a dispersão do propágulo quando os níveis de água estão mais elevados.

Nas áreas de resíduos sólidos e desmatamento, Avicennia spp. apresentou altos índices de sincronia de floração. Esse alto índice de sincronismo entre os indivíduos de uma mesma espécie pode favorecer a polinização cruzada devido ao aumento na atração de polinizadores.

Na área de baixo impacto, as fenofases reprodutivas de Rhizophora mangle apresentaram alto sincronismo. O fato de Rhizophora mangle não ter apresentado o mesmo padrão nas áreas impactadas pode sugerir que ela seja mais sensível aos impactos em estudo.

McKee et al. (2002) consideram a espécie extremamente plástica em sua demanda de nutrientes e tolerância à salinidade. Entretanto, em relação aos aspectos fenológicos, já foi observado que a maior produção de flores ocorre quando há maior disponibilidade de nutrientes e a salinidade é relativamente baixa (AKÉ-CASTILLO; VÁZQUEZ; LÓPEZ-PORTILLO, 2006; AGRAZ HERNÁNDEZ et al., 2015).

Dessa forma, se os impactos alteram esses fatores abióticos, essas alterações irão interferir de maneira direta na fenologia dessa espécie. Agraz Hernández et al. (2015) afirmam que $R$. mangle é muito sensível a mudanças na disponibilidade de nutrientes, oxigênio dissolvido na água subterrânea, salinidade e no hidroperíodo, principalmente quando comparada a outras espécies

Os métodos fenológicos analisados neste estudo forneceram dados essencialmente diferentes. Os percentuais de intensidade para floração e frutificação de Avicennia spp., Laguncularia racemosa e Rhizophora mangle são em sua maioria inferiores aos percentuais de atividade; isso significa que há alta sincronia entre os 
indivíduos dessas espécies, porém baixa intensidade de floração. Em virtude disso, os picos de atividade e intensidade nem sempre coincidiram para as três espécies de mangue amostradas. Os estudos apresentados por Bencke e Morellato (2002a) e Matni (2007) apontaram que a proporção de indivíduos em atividade nem sempre prediz claramente o real volume de flores e frutos produzidos, ressaltando assim a importância da conjugação dos dois métodos. A combinação de métodos em estudos fenológicos também é recomendada por Morellato et al. (2010).

Por meio do índice de atividade pode-se revelar o período em que a floração e a frutificação das espécies de interesse manifestaram atividade de forma mais generalizada nas populações, observando melhor do que com percentual de intensidade o início e o final da atividade em cada área de estudo. Os picos de intensidade apontaram quando a floração e a frutificação foram mais intensas nos grupos pesquisados, considerando a quantidade estimada na produção de flores e frutos e não apenas o número de indivíduos manifestando a fenofase (MATNI, 2007).

Os percentuais de intensidade foram menos expressivos que os de atividade neste trabalho, isso pode estar relacionado a fatores bióticos como polinizadores e dispersores, já que a atividade e abundância destes estão correlacionadas diretamente com a disponibilidade dos recursos florais e frutíferos (BENCKE; MORELLATO, 2002b). Uma vez que se observou que os fatores abióticos como umidade, salinidade, temperatura e pluviosidade não apresentaram influência direta sobre os resultados.

De acordo com o nível de análise (intrapopulacional ou interpopulacional), foi observado presença de comportamento fenológico distinto (FERNANDES et al., 2005; MATNI, 2007). Este pode representar diferentes estratégias ecológicas relacionadas às características particulares de cada espécie em busca de favorecer a dispersão, o estabelecimento e o recrutamento de plântulas (NADIA; MORELLATO; MACHADO, 2012). Com base nos resultados obtidos nesta pesquisa, observa-se que embora o índice de atividade forneça informações distintas dos encontrados pela utilização do índice de intensidade de Fournier (1974) sobre o comportamento fenológico, ele 
auxilia na análise e interpretação dos padrões encontrados em cada população, uma vez que combina dados de sincronia e intensidade.

A ausência de correlação com os fatores abióticos também foi observado por Matni (2007). Neste estudo a falta de correlação na área de baixo impacto sugere que os impactos analisados nesta pesquisa provavelmente exercem maior força seletiva sobre os percentuais de atividade e intensidade da floração e frutificação que os fatores abióticos avaliados. Os manguezais apresentam complexidade funcional e altas taxas de resiliência e resistência. Mesmo assim, a estrutura e fisiologia dos seus componentes podem sofrer alterações em resposta a mudanças ambientais (DA SILVA; DA SILVA; SILVA ARAÚJO, 2019) ficando mais sensíveis a certos impactos negativos (Tabela 1).

Respostas endógenas da planta e pressões seletivas bióticas devem influenciar a periodicidade das fenofases de algumas espécies deste estudo. Características filogenéticas podem iniciar os ciclos reprodutivos em detrimento das condições climáticas (FORTUNATO; QUIRINO, 2016).

Rizhopora mangle tem sido apontada como uma espécie de floração contínua, porém com o aumento no processo de produção atreladas, principalmente, ao período chuvoso (MENEZES, 2010; AGRAZ HERNÁNDEZ et al., 2015). Entretanto neste trabalho o pico de floração e frutificação ocorreu dentro dos meses mais secos, diferente também do que foi apresentado por Matni (2007), que não observou diferenças significativas quanto à produção das fenofases estudadas no mangue da península de Ujuruteua, Pará. Estudo realizado por Cuzzuol e Campos (2001) dá indicação que as espécies Laguncularia racemosa e Rhizophora mangle são dotados de mecanismos fisiológicos osmorregulatórios complexos em relação aos elevados teores salinos a que estão submetidos constantemente como controle de potenciais iônicos através da membrana, atividade enzimática, por exemplo. Adaptações morfo-fisiologicas podem estar atuando como facilitadores do sucesso reprodutivos destas espécies. 
A capacidade de propagação vegetativa das espécies de mangue é limitada (CARDOSO; CHAVES; SOARES, 2015), portanto, entender como se dá o comportamento da planta em relação a sua biologia reprodutiva é importante, especialmente utilizando-se desta informação de como os impactos estão interferindo nos processos fenológicos, tendo em vista que a regeneração de manguezais degradados depende, em parte, da dispersão, do aporte de propágulos e da sobrevivência de suas plântulas.

Quanto mais se conhece sobre os padrões dentro dos ecossistemas, melhores são as chances de se elaborar planos de manejo que irão subsidiar futuras ações de monitoramento e conservação.

\section{CONCLUSÕES}

Ao se analisar os padrões fenológicos de floração e frutificação das três espécies sujeitas a diferentes graus de perturbação antrópica, concluiu-se que as alterações resultantes das atividades humanas, direta ou indiretamente, afetam o comportamento reprodutivo e produtivo do mangue.

As florações de Avicennia spp. e Rhizophora mangle, em condições naturais, ocorrem o ano todo. Nas áreas impactadas essas espécies mostraram padrões irregulares.

Já em relação à frutificação, Laguncularia racemosa foi a espécie que apresentou os mais altos percentuais para os dois índices fenológicos analisados. Rhizophora mangle frutificou o ano todo na área de baixo impacto. Os índices de frutificação da Avicennia spp. foram inferiores quando comparados aos seus percentuais de floração.

Os fatores abióticos como pluviosidade e salinidade parecem exercer maior força seletiva quando as espécies estão sujeitas a algum tipo de impacto antrópico.

A análise comparativa dos diferentes métodos de avaliaçãofenológicos realizados neste estudo indicou que estes fornecem dados distintos e complementares sobre o comportamento reprodutivo vegetal e de como os impactos a que estes ambientes estão sujeitos pode estar interferindo negativamente sobre a flora deste ecossistema. 


\section{REFERÊNCIAS}

AGRAZ HERNÁNDEZ, C. M. et al. Phenological variation of Rhizophora mangle and ground water chemistry associated to changes of the precipitation. Hidrobiológica, México, v. 25, n. 1, p. 49-61, 2015.

AKÉ-CASTILLO, J. A.; VÁZQUEZ, G.; LÓPEZ-PORTILLO, J. Litterfall e decomposição de Rhizophora mangle L. em uma lagoa costeira no sul do Golfo do México. Hidrobiology, [s. I.], n. 559, p. 101-111, 2006.

ALVARENGA, A. M. S. B.; BOTOSSO, P. C.; SOFFIATTI, P. Stem growth and phenology of three subtropical mangrove tree species. Brazilian Journal of Botany, São Paulo, v. 40, n. 4, p. $907-$ 914, 2017.

BENCKE, C. S. C.; MORELLATO, L. P. C. Comparação de dois métodos de avaliação da fenologia de plantas, sua interpretação e representação. Brazilian Journal of Botany, São Paulo, v. 25, n. 3, p. 269-275, 2002a. DOI: http://dx.doi.org/10.1590/S0100-84042002000300003.

BENCKE, C. S. C. MORELLATO, L. P. C. Estudo comparativo da fenologia de nove espécies arbóreas em três tipos de floresta atlântica no sudeste do Brasil. Brazilian Journal of Botany, São Paulo, v. 25, n. 2, p. 273-248, jun. 2002b. DOI: http://dx.doi.org/10.1590/S010084042002000200012.

BONALDI, R. A.; RODERJAN, C. V. Levantamento florístico e caracterização estrutural de um manguezal na APA de Guaraqueçaba, Paranaguá, PR. Acta Biológica Catarinense, [s. I.], v. 4, n. 1, p. 19-28, jan./jun, 2017.

CARDOSO, C. S.; CHAVES, F. O.; SOARES, M. L. G. Variação espaço-temporal na produção de propágulos de espécies de mangue no Sudeste do Brasil. Boletim do Museu Paraense Emílio Goeldi - Ciências Naturais. Belém, v. 10, n. 3, p. 491-503, set./dez. 2015.

CUZZUOL, G. R. F.; CAMPOS, A. Aspectos nutricionais na vegetação de manguezal do estuário do Rio Mucuri, Bahia, Brasil. Brazilian Journal of Botany, São Paulo, v. 24, n. 2, p. 227-234, jun. 2001.

FERNANDES, M. E. B. et al. Padrões de floração e frutificação em Laguncularia racemosa (L.) Gaertn. F.: uma avaliação metodológica. Boletim do Laboratório de Hidrologia, [s. I.], v. 18, p. 33-38, 2005.

FERREIRA, A. C.; BEZERRA, L. E. A.; MATTHEWS-CASCON, H. Aboveground carbon stock in restored neotropical mangrove: influence of management and brachyuran crab assemblage. Wetlands Ecology and Management, Dordrecht, v. 27, n. 2, p. 233-242, 2019.

FERREIRA, M. C.; CONSOLARO, H. Fenologia e síndromes de polinização e dispersão de espécies de sub-bosque em um remanescente florestal urbano no Brasil central. Bioscience Journal, Uberlândia, v. 29, n. 5, p. 1708-1720, nov, 2013.

FORTUNATO, M. E. M.; QUIRINO, Z. G. M. Efeitos da fragmentação na fenologia reprodutiva de espécies arbóreas presentes em borda e interior de Mata Atlântica Paraibana. Rodriguésia, Rio de Janeiro, v. 67, n. 3, p. 603-614, 2016. 
FOURNIER, L. A. Un método cuantitativo para la medición de características fenológicas em árboles. Turrialba, San José, n. 24, p. 422-423, 1974.

FUNDAÇÃO CEARENSE DE METEOROLOGIA E RECURSOS HÍDRICOS. Posto Pluviométrico de Acaraú. Fortaleza, 2016. Disponível em: http://www.funceme.br. Acesso em: 01 set. 2017.

LIMA, K. O. O. Dinâmica Populacional de plântulas de Rhizophora mangle L. no sudeste do Brasil. 2018. Tese (Doutorado) - Universidade Federal do Espírito Santo, Centro de Ciências Humanas e Naturais, Espírito Santo, 2018.

MATIAS, L.; SILVA, M. D. Monitoramento e análise da vegetação de manguezal no litoral sul de Alagoas. Journal of Environmental Analysis and Progress, [s. I.], v. 2, n. 3, p. 312-319, 2017.

MATNI, A. S. Estudo comparativo da fenologia reprodutiva de Rhizophora mangle L, Avicennia germinans (L.) Stearn e Laguncularia racemosa (L.) Gaertn. F. ao longo da península de Ajuruteua, Bragança - Pará. 2007. Dissertação (Mestrado em Biologia Ambiental) - Universidade Federal do Pará, Bragança, 2007.

MCKEE, K. L. et al. Mangrove isotopic (d15N and d13C) fractionation across a nitrogen vs. phosphorus limitation gradient. Ecology, [s. I.], v. 83, n. 4, p. 1065-1075, 2002.

MENEZES, L. C. S. Estrutura e produção de serapilheira de floresta de mangue da região estuarina-lagunar do baixo São Francisco sergipano. 2010. Dissertação (Mestrado) Universidade Federal de Sergipe, São Cristóvão, 2010.

MORELLATO, L. P. C. et al. Estratégias fenológicas de espécies arbóreas em floresta de altitude na Serra do Japi, Jundiaí, SP. Revista Brasileira de Biologia, São Carlos, n. 50, p. 149-162, 1990.

MORELLATO, L. P. C. et al. The Influence of Sampling Method, Sample Size, and Frequency of Observations on Plant Phenological Patterns and Interpretation in Tropical Forest Trees. In: HUDSON I.; KEATLEY M. (ed.). Phenological research. Dordrecht: Springer, 2010. p. 99-121.

NADIA, T. L.; MORELLATO, L. P. C.; MACHADO, I. C. Reproductive phenology of a northeast Brazilian mangrove community: environmental and biotic constraints. Flora-Morphology, Distribuition, Functional Ecology of Plants, [s. I.], v. 207, n. 9, p. 682-692, 2012.

OCHOA-GAONA, S.; HERNÁNDEZ, I. P.; JONG, B. H. J. Fenología reproductiva de las especies arbóreas del bosque tropical de Tenosique, Tabasco, México. Revista de Biologia Tropical, Costa Rica, v. 56, n. 56, p. 657-673, 2008.

PAULA, A. L. S. et al. The recovery of a degraded mangrove in Ceará through the Production of Laguncularia racemosa (L.) C.F. Gaertn. (Combretaceae) and Avicennia sp. Stapf ex Ridl (Acanthaceae) seedlings. Revista Árvore, Viçosa, MG, v. 40, n. 3, p. 377-385, 2016.

SILVA, A. P.; SILVA, J. B.; ARAÚJO, E. D. S. Marisma, Manguezal (Mangue e Apicum): Ecossistema de transição terra-mar do Brasil. Revista Brasileira de Geografia Física, [s. I.], v. 13, n. 2, p. 727-742, abr. 2020.

SOUSA, W. P. et al. Supply-side ecology in mangroves: do propagule dispersal and seedling establishment explain forest structure? Ecological Monographs, Washington, n. 77, p. 53-76, 2007. 


\section{Contribuição de Autoria}

\section{1 - Jorgeana de Almeida Jorge Benevides}

Licenciada em Biologia, Doutoranda, Professora

https://orcid.org/0000-0003-0997-8331•jorgeana.benevides@ifce.edu.br

Contribuição: Administração do projeto, Conceituação, Curadoria dos dados, Metodologia, Visualização dos dados, Investigação, Escrita - primeira redação, Escrita - revisão e edição

\section{2 - Rafaela Camargo Maia}

Bióloga, Dra., Professora

https://orcid.org/0000-0001-5871-4610•rafaelamaia@ifce.edu.br

Contribuição: Administração do projeto, Conceituação, Curadoria dos dados, Metodologia, Escrita - revisão e edição

\section{3 - Ingrid H'Oara Carvalho Vaz da Silva}

Licenciada em Biologia, Doutoranda, Professora

https://orcid.org/0000-0002-0436-6099•ingrid.hoara@ifce.edu.br

Contribuição: Conceituação, Investigação, Metodologia, Escrita - revisão e edição

\section{Como citar este artigo}

Benevides, J. A. J.; Maia, R. C.; Silva, I. H. C. V. Monitoramento fenológico para avaliação de impacto ambiental em manguezais estuarinos no Nordeste do Brasil. Ciência Florestal, Santa Maria, v. 31, n. 4, p. 1631-1653, 2021. DOI 10.5902/1980509839683. Disponível em: https://doi. org/10.5902/1980509839683. 